adenylate cyclase-activating polypeptide and growth hormone-releasing hormone-like peptide in the frog, Rana ridibunda. J Comp Neurol 2000 ; 421 : 234-46.

7. McRory JE, Parker RL, Sherwood NM. Expression and alternative processing of a chicken gene encoding both growth hormone-releasing hormone and pituitary adenylate cyclase-activating polypeptide. DNA Cell Biol 1997 ; 16: 95-102.

8. Vaudry D, Gonzalez BJ, Basille M, et al. Pituitary adenylate cyclase-activating polypeptide and its receptors: from structure to functions. Pharmacol Rev $2000 ; 52: 269-324$.

9. Parker DB, Power ME, Swanson P, et al. Exon skipping in the gene encoding pituitary adenylate cyclaseactivating polypeptide in salmon alters the expression of two hormones that stimulate growth hormone release. Endocrinology 1997 ; 138 : 414-23.

10. Montero M, Yon L, Kikuyama S, et al. Molecular evolution of the growth hormone-releasing hormone/ pituitary adenylate cyclase-activating polypeptide gene family. Functional implication in the regulation of growth hormone secretion. J Mol Endocrinol 2000 ; $25: 157-68$.

11. Lee LT, Siu FK, Tam JK, et al. Discovery of growth hormone-releasing hormones and receptors in nonmammalian vertebrates. Proc Natl Acad Sci USA $2007 ; 104: 2133-8$.

12. Conlon JM, Larhammar. The evolution of neuroendocrine peptides. Gen Comp Endocrinol 2005; $142: 53-9$.

\title{
NOUVELLE
}

\section{WTX, un nouveau gène suppresseur de tumeur muté dans la tumeur de Wilms}

\author{
Stéphane Angers
}

\author{
Chaire de recherche du Canada en architecture \\ fonctionnelle des complexes de signalisation, \\ Leslie Dan Faculty of Pharmacy, University of Toronto, \\ Toronto, Ontario, M5S 3M2 Canada.
} stephane.angers@utoronto.ca
> La tumeur de Wilms est un cancer rénal affectant principalement les enfants [1]. Chez $15 \%$ de ces patients, les tumeurs contiennent des mutations du gène suppresseur de tumeur WTl et de la $\beta$-caténine, la protéine centrale de la voie de signalisation Wnt. Malgré de nombreuses études, les gènes supplémentaires qui pourraient contribuer à l'étiologie de ce cancer tardent à être identifiés. Récemment, la protéine WTX, qui n'avait jusqu'alors aucune fonction connue, a été suspectée car son expression est éteinte dans $30 \%$ des tumeurs de Wilms [2]. Indépendamment, au cours de notre travail sur la voie de signalisation Wnt, nous avons aussi identifié WTX et démontré que cette protéine était importante pour le contrôle de l'activité de la $\beta$-caténine [3]. Ces travaux offrent donc une base moléculaire pour la compréhension du mode de fonctionnement normal de WTX, mais également pour celle de son dysfonctionnement lorsqu'elle est mutée dans la tumeur de Wilms.

\section{Voie de signalisation Wnt \\ et cancers humains}

Conservée au cours de l'évolution, la voie de signalisation Wnt- $\beta$-caténine contrôle plusieurs aspects du développement embryonnaire ainsi que l'homéos- tasie cellulaire dans plusieurs tissus chez l'adulte [4, 5]. À l'état basal, la protéine $\beta$-caténine est constitutivement entrầnée vers le processus d'ubiquitinylation, avec pour conséquence sa destruction par le protéasome. Les protéines APC (adenomatous polyposis coli), Axine, Caséine kinase la et GSK-3b (glycogen synthase kinase 3 beta) font partie du même complexe protéique appelé le complexe de destruction. Le complexe de destruction facilite la phosphorylation de la $\beta$-caténine, un signal qui est requis pour son recrutement vers la machinerie d'ubiquitinylation. Lorsque cette voie de signalisation est activée par la liaison du ligand Wnt aux récepteurs Frizzled et LRP5/6 à la surface de la cellule, le complexe de destruction est inhibé, ce qui entraîne la stabilisation de la $\beta$ caténine. Cette accumulation soudaine de $\beta$-caténine permet son cheminement vers le noyau où elle pourra réguler l'expression d'un programme génique qui mènera à la réponse biologique désirée. Plusieurs cancers chez l'humain sont associés à des mutations des protéines du complexe de destruction ou, encore plus directement, au niveau des sites de phosphorylation de la $\beta$-caténine qui sont ciblés par les kinases de ce complexe. Dans ces cancers, il y a une accumulation nucléaire de $\beta$-caténine, et par conséquent une hyperactivation de la voie Wnt qui résulte en l'activation incontrôlée de la prolifération cellulaire. Toutefois dans d'autres cancers, dont la tumeur de Wilms, l'accumulation nucléaire de la $\beta$-caténine est parfois observée, mais elle ne s'accompagne pas de mutations causatives connues des membres identifiés du complexe de destruction ou de la $\beta$-caténine elle-même. Bien qu'une activation constitutive de la voie Wnt puisse découler du dysfonctionnement de protéines agissant en amont du complexe de destruction, ou encore de défauts épigénétiques entraînant la surexpression des ligands Wnt ou la réduction de différents régulateurs négatifs, il est plausible de faire l'hypothèse selon laquelle des protéines supplémentaires participent à la régulation de la stabilité de la $\beta$-caténine au niveau du complexe de destruction.

\section{Identification de la protéine WTX comme partenaire de la voie de signalisation Wnt}

Avec l'objectif d'identifier ces régulateurs supplémentaires, nous avons entrepris une étude systématique des complexes protéiques formant le complexe de destruction de la $\beta$-caténine. 
Notre approche a consisté en la purification biochimique des différentes protéines formant le complexe de destruction à partir de cellules humaines, suivie de l'analyse de leur composition par spectroscopie de masse. L'analyse itérative de plusieurs membres connus du complexe de destruction nous a permis d'établir une architecture détaillée des interactions protéines-protéines survenant entre les différents membres du complexe et dont on pouvait prédire qu'elles étaient requises pour son fonctionnement [3]. Nous avons logiquement identifié tous les membres connus du complexe de destruction de la $\beta$-caténine, mais nous avons aussi découvert que la protéine WTX s'associait à toutes les protéines de ce complexe que nous avons examinées. Ces résultats posi-

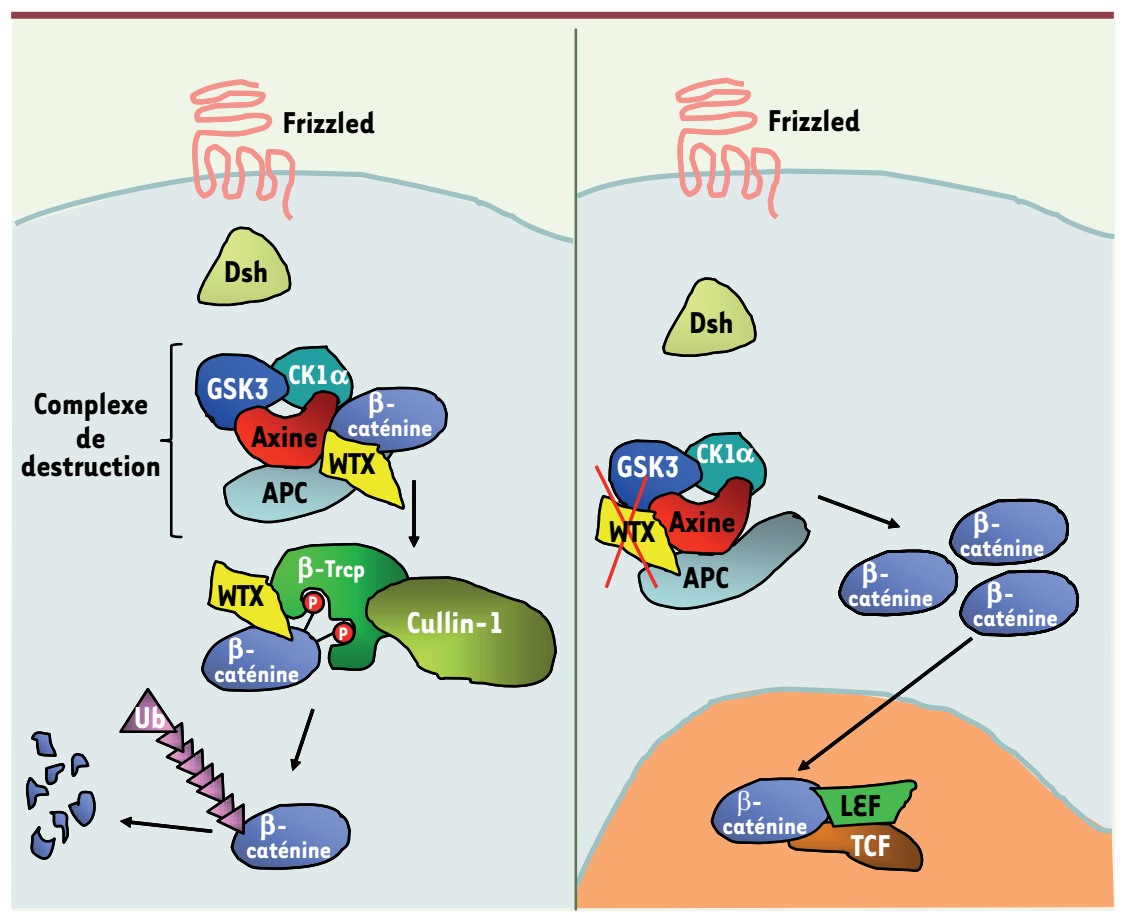

Figure 1. Rôle joué par WTX dans le complexe de destruction de la $\beta$-caténine. A. À l'état basal, le complexe de destruction (APC, Axine, GSK3b, CKl $\alpha$ ) catalyse la phosphorylation de la $\beta$-caténine. Phosphorylée, la $\beta$-caténine devient un substrat de haute affinité pour une $\varepsilon 3$ ubiquitine ligase de type SCF (Skpl, F-Box [ $\beta$-Trcp], Culline-1) qui entraîne sa dégradation. WTX est retrouvée dans les complexes protéiques d'Axine, d'APC, de $\beta$-caténine et de $\beta-\operatorname{TrCP}$ soulevant la possibilité que WTX puisse agir au niveau de la phosphorylation ou de l'ubiquitinylation de la $\beta$ caténine. $B$. Dans les tumeurs de Wilms, WTX est mutée, ce qui compromet le bon fonctionnement du complexe de destruction et entraîne l'accumulation de la $\beta$-caténine. La $\beta$-caténine est alors transloquée constitutivement dans le noyau où, en se liant aux protéines Lef et Tcf, elle pourra modifier l'expression de gènes.
WTX, comme les autres membres du complexe de destruction, contrôle la stabilité protéique de la $\beta$-caténine. À l'appui de cette hypothèse, nous avons démontré que lors de la reconstitution biochimique de l'activité du complexe de destruction en utilisant des extraits d'oocytes de Xénope, l'ajout de protéines WTX purifiées accélérait la cinétique d'ubiquitinylation et de dégradation de la $\beta$-caténine [3]. Des expériences supplémentaires de gain et de perte de fonction dans les embryons de xénope et de poisson-zèbre, deux modèles classiques d'analyse de la voie de signalisation Wnt, ont confirmé ces résultats et ont permis de démontrer que WTX exerçait ce rôle de régulateur négatif dans le contexte d'un animal multicellulaire en développement [3].

\section{Perspectives}

D'autres efforts sont maintenant nécessaires pour mieux comprendre le mode d'action précis de WTX. WTX agitelle de concert avec l'Axine et les kinases du complexe de destruction pour phosphoryler la $\beta$-caténine? Puisque WTX interagit aussi avec $\beta$-TrCP, la protéine adaptatrice qui recrute la $\beta$-caténine vers la machinerie d'ubiquitinylation, participe t-elle plutôt à la réaction d'ubiquitinylation?

Étonnamment, WTX est le premier gène suppresseur de tumeur situé sur le chromosome $X$. Puisque seulement I'un des deux chromosomes $X$ est actif dans les cellules somatiques, la mutation d'un gène présent sur ce chromosome pourrait être suffisante pour l'initiation ou la progression de tumeurs. Ce n'est pas le cas pour la majorité des autres gènes suppresseurs de tumeur où deux événements indépendant sont requis pour I'inactivation de chaque allèle. WTX représente donc un troisième membre du complexe de destruction qui est fonctionnellement inactivé dans différents cancers humains. On connaît les mutations d'APC et de l'Axine dans le cancer du côlon. Des mutations 
de WTX pourraient-elles être détectées dans d'autres cancers que les tumeurs de Wilms, incluant le cancer du côlon, et, inversement, des mutations d'APC et de l'Axine seront-elles détectées dans les tumeurs de Wilms? On ne peut pas exclure qu'il y ait une spécificité fonctionnelle de chaque gène suppresseur de tumeur dont l'importance serait fonction du contexte cellulaire. Comme c'est le cas pour chaque nouvelle percée scientifique, l'identification de WTX comme un nouveau gène suppresseur de tumeur modulant la voie de signalisation Wnt soulève maintenant plusieurs questions, mais contribue simultanément à une meilleure compréhension des mécanismes moléculaires régissant le cancer. $\diamond$

The tumor suppressor gene WTX, mutated in Wilms tumours, is $\alpha$ member of the $\beta$-catenin destruction complex

\section{RÉFÉRENCES}

1. Rivera MN, Haber DA. Wilms' tumour: connecting tumorigenesis and organ development in the kidney. Nat Rev Cancer $2005 ; 5:$ 699-712.

2. Rivera MN, Kim WJ, Wells J, et al. An X chromosome gene, WTX, is commonly inactivated in Wilms tumor. Science 2007 ; 315 : 642-5

3. Major MB, Camp ND, Berndt JD, et al. Wilms tumor suppressor WTX negatively regulates WNT/betacatenin signaling. Science 2007 ; 316 : 1043-6.

4. Wodarz A, Nusse R. Mechanisms of Wnt signaling in development. Annu Rev Cell Dev Biol 1998; 14 : 59-88.

5. Moon RT, Kohn AD, De Ferrari GV, Kaykas A. WNT and beta-catenin signalling: diseases and therapies. Nat Rev Genet 2004 ; 5 : 691-701.

\section{NOUVELLE}

\section{Un retournement de situation à l'origine des vertébrés}

Guillaume Balavoine
Centre de Génétique Moléculaire, CNRS, Avenue de la Terrasse, 91198 Gif-sur-Yvette, France. guillaume.balavoine@cgm.cnrs-gif.fr

apportent un soutien décisif à l'hypothèse du retournement [5]. Cette équipe s'intéresse à la génétique du développement de l'architecture du système nerveux, c'est-à-dire aux gènes qui, au cours de l'embryogenèse, vont, par leur expression différenciée dans les tissus précurseurs du système nerveux, déterminer sa forme et l'agencement des nombreux types de neurones qu'il comprend. On sait que, chez les vertébrés, le développement du système nerveux est très particulier puisque le cerveau et la moelle épinière proviennent de la différenciation d'un tube neural qui se forme sur toute la longueur de l'animal par le rapprochement et la fusion de replis latéraux de l'ectoderme dorsal de l'embryon. Dès avant cette fusion, une batterie de gènes régulateurs appartenant notamment aux familles des gènes à homéoboîtes NK et Pax est exprimée en une série de colonnes longitudinales symétriques et intervient pour spécifier les différents types de cellules neurales qui vont se différencier le long de ce tissu nerveux, notamment des neurones moteurs à proximité de la face ventrale du tube, et des neurones sensoriels en position dorsale. 